\title{
3D Virtual Vision Construction Platform for Airport Design Evaluation Based on Virtual Reality Technique
}

\author{
Zuo Ling ${ }^{1}$, He Yong ${ }^{1}$, Wang Lan $^{2}$ and Zhu Xinping ${ }^{2+}$ \\ ${ }^{1}$ East China Regional Air Traffic Management Bureau of Civil Aviation of China, Shanghai, China, 200335 \\ ${ }^{2}$ College of Air Traffic Management, Civil Aviation Flight University of China, Guanghan Sichuan, China, \\ 618307
}

\begin{abstract}
Airport is the start and end node of the air transportation system. The development of simulation platform for the evaluation of airport expansion or building scheme has become a common concern of the industry. In this paper, a 3-dimensional virtual vision fast construction platform for airport design evaluation was developed based on VR technology. Typical models related to elements of airport airside area has been produced and integrated into the platform, including the static ones (such as runway, taxiway) and movement objects (aircraft and vehicle). The weather effect models were incorporated into the platform. Airport 3D virtual vision model could be quickly and efficiently created by selecting models and modify the parameters of models in this platform. The plug-in technology is used in the development of the platform, which is contribute to the integration of evaluation algorithms for airport planning and design scheme. The VR technology used in this platform provides an intuitive means to verify the airport design schema through the VR helmet and handle. As a demonstration, we have developed an air traffic control tower position sitting function package. The test result indicates that the platform meets the requirement of reliability on creating $3 \mathrm{D}$ virtual vision, and it is easy-use to airport designers.
\end{abstract}

Keywords: airport; design evaluation; virtual vision; virtual reality

\section{Introduction}

Airport is the start and end node of the whole air transport system. An airport system with favourable design is important for the promotion of safety and efficiency of the air transport system. In recent years, the demand for the new building and expansion of civil airports is emerging. How to establish a professional simulation platform and how to verify the rationality of the airport building or expansion scheme has become a concern of the industry. In this paper, the Virtual Reality (VR) technique is adopted to develop a threedimensional virtual vision fast construction platform, which provides a mean to produce a model of airport airside quickly and efficiently. This platform also could provide a mean to integrate some evaluation algorithms for airport build or expansion plan. Thus, we could carry out 'what-if' test to verify the operational performance of different airport airside designs in the platform.

Virtual Reality (VR) is based on computer technology, combined with other related technologies, to produce a digital environment that is highly similar to a real environment in sight, hearing, and touch. Users could interact with objects in the virtual digital environment or with each other by necessary equipment, acquiring good experience of being in the real environment [1]. After years of development, VR technology has been used in national defence and military [2], aerospace [3], and manufacturing industry [4], and so on. In the field of civil aviation, it is used in virtual ATC (Air Traffic Control) tower system [5], civil aircraft flight management system [6], etc. In addition, Durham University in UK firstly proposed the idea of using computer game engines to build virtual environment prototypes, and a fire evacuation virtual drill system [7,

\footnotetext{
+ Corresponding author. Tel.: + 86-13419037831; fax: +86-0838-5190242.

E-mail address: zhu408@163.com
} 
8]. Researchers at Dartmouth College in the United States used game engines to realize the virtual training scene of plane crash which caused many casualties, and built a web-based training system [9], and carried out the research of building the ground feature model, character model and behaviour simulation [10].Generally speaking, the VR technology has a good prospect on its employment in civil aviation.

In this paper, a method is proposed to establish a platform integrating general 3-dimensional models for elements in the airport airside area, and the complex airside traffic system could be quickly recreated which is very similar to the real environment in airport airside area. Additionally, the plug-in technology is used in this platform, which supports the dynamic integration of evaluation algorithms for airport planning and design scheme.

\section{Function Design for 3D Virtual Vision Fast Construction Platform of Airport}

The main features of this platform are as follows:

(1) The platform supports the dynamic integration and management of airport basic model element, like runway, taxiway, apron stands or other ground marking, etc. And, the simple building models for terminal, air-bridge, auxiliary building, and approach light could be stored in the platform. By this platform, we can select from the model library and import 3D model elements to generate the virtual visual corresponding to the airport design schema, such as simple model for terminal, approach light, and so on.

(2) The platform supports parameter modification of model imported by user. Due to different airport construction or expansion plans, we need to generate the closely similar virtual 3D visual. The platform includes tools to modify the property parameter of the models. For example, we can change the height or width of the terminal building, or the position of the ATC tower. Fatherly, the customized weather effect displays including day-night, sunny, cloud can be modified in this platform.

(3) The platform integrates numbers of aircraft and vehicle 3D models for different aircraft type or airlines. And we could flexibly modify the taxiing routes for aircrafts or vehicles in the platform, fulfilling the VR simulation of airport surface operation process, such as the aircraft pushback or taxiing.

(4) The plug-in technology is used in this platform, which supports the dynamic integration of evaluation algorithms for airport planning and design scheme.

\section{The Software Architecture Design of the Platform}

The 3-dimensional virtual vision fast construction platform for the airport airside area adopts the following development process:

Firstly, we need to extract the main environmental characteristics of the airport airside area scene. The 3DMax software is employed to create 3-dimensional model required for the creation of airside area visual, including ordinary object model, such as terminal, ATCT, auxiliary building, etc. And, the ground model elements, such as runways, taxiways, aprons, stop bars and other ground markings, were created to forms a model library. Additionally, numbers of aircrafts and vehicle with different airline logo were created. All of the models will be imported into the Unity3D engine for model binding.

Secondly, the software architecture of the platform for 3D virtual visual simulation is developed by Unity3D software. And the models in model library can be managed in a centralized mode. At the same time, the software module for visual generation was developed. By this module, we could create a 3D airport visual models by selecting models from the model library and modify the parameters of the model.

Thirdly, the Unity3D engine is used to develop the airside area simulation function module, fulfilling the aircraft or vehicle movement simulation. And this module supports the interaction between users and visual by personal computer or VR smart helmet. Thus, the avatar could travel in the virtual 3D visual.

Finally, the plug-in function modules could be developed according to the evaluation object. In this paper, we realized a function package for ATCT position sitting according to the approved national ATCT design standards and guidelines.

The software architecture design of the platform is demonstrated in Fig. 1. 


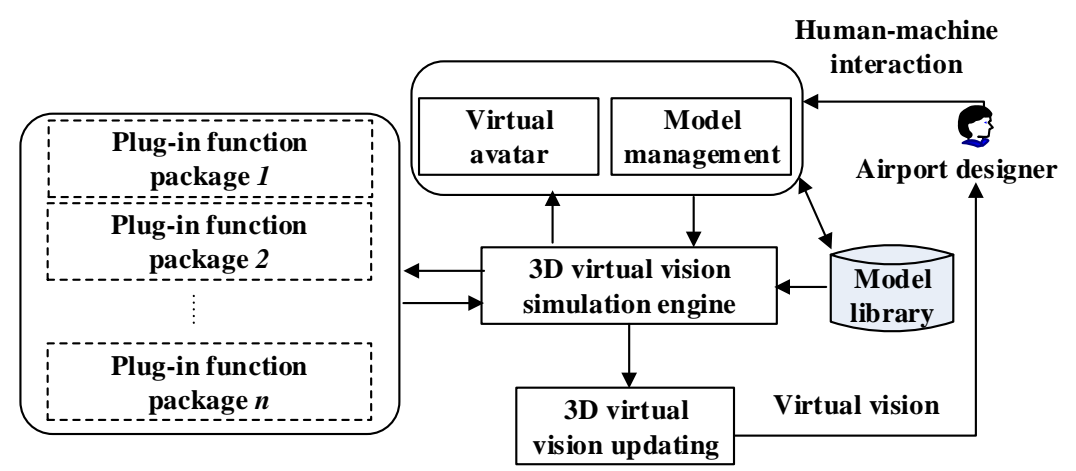

Fig. 1: software architecture of the platform for $3 \mathrm{D}$ virtual vision fast construction

For an airport design needed to be verified, we could adopt the platform. First of all, based on the airside area environment of the airport design, we select the corresponding model from the model library and set the model parameters (such as setting the height of the tower and the height of the terminal building). And then, the model management function module can be used to set the object movement logic on airport surface, for example, the pushback routes, taxi-in or taxi-out route of aircraft. Additionally, based on the simulation engine we developed, a 3-dimensional virtual visual environment could be created by models we selected. Finally, according to the evaluation object, we could select plug-in algorithm function module integrated in the platform, and demonstrate the virtual vision and quantitative results. At present, this platform integrates the ATCT position sitting function package.

\section{Key Features of the Platform for 3D Virtual Airport Vision Fast Construction}

\subsection{D Model for Airside Area Elements}

The platform for 3D virtual airport visual fast construction is mainly developed for airport airside design evaluation. Thus, the 3D models were mainly developed for this area, including conventional static simple models (such as terminal, auxiliary building, air traffic control tower, etc.), active surface target models (such as vehicles, aircrafts). All of these models were stored and managed by model library. In this paper, the 3DMax software is adopted to produce 3-dimensional models and then these models were imported into Unity3D software engine in .fbx format. The Unity3D engine can complete the rendering of threedimensional virtual vision, including the load of static and active models, and support users to complete roaming in the virtual vision. Typical 3D simple building model and aircraft model in virtual vision are shown in Fig. 2 and Fig. 3.

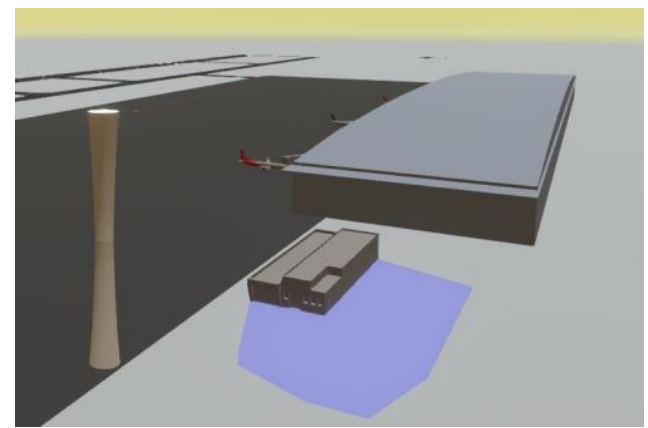

Fig. 2: 3D simple building model in virtual vision

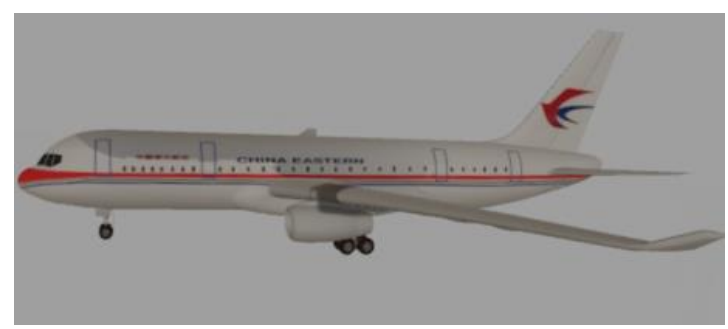

Fig. 3: 3D aircraft model in virtual vision 


\subsection{Creation of 3D Virtual Vision}

The development of 3D virtual vision creation function was one of the main modules in this platform. The 3D virtual vision of the airport airside area was generated based on the model selected and set by the user. Meanwhile the vision is dynamically updated as the virtual form roams during the virtual simulation process. The construction process of the virtual scene is shown in Fig. 4. Users can extract models from the model library and set the vision according to the modelling needs in the parameter setting interface. Users also can set the attributes of model, such as setting the length of the runway, changing the height of the tower, and changing the taxi route of the aircraft, as shown in Fig. 5.

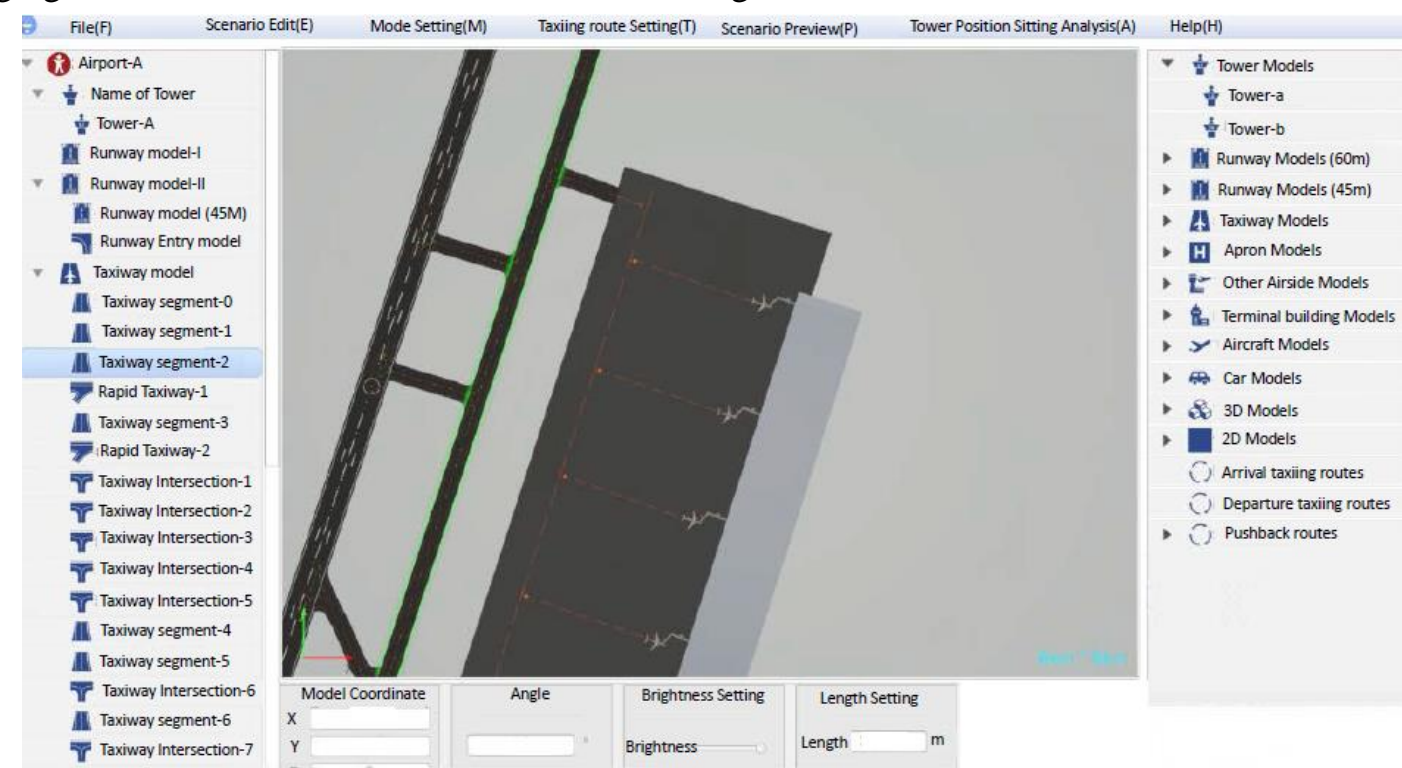

Fig. 4: Platform interface for the virtual construction by using model library

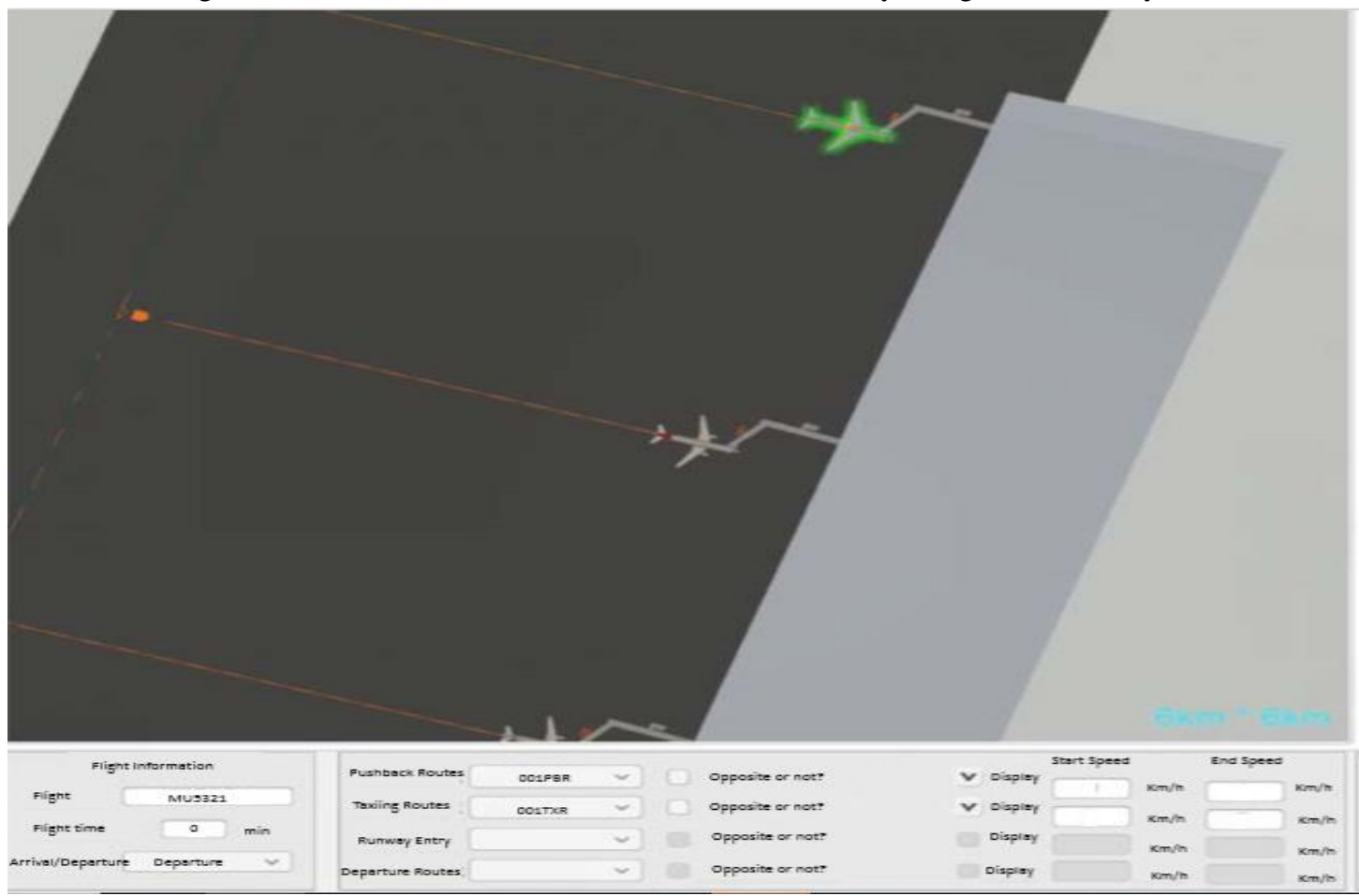

Fig. 5: Parameter setting interface for models

The vision update process can be achieved by Unity3D script in this platform. And the Unity3D's own physics engine was adopted to rendering better vision effects, such as collision and motion of surface objects. Meanwhile, taking into account the user's roaming in the $3 \mathrm{D}$ virtual vision, sound effects were also introduced into the vision, and human-computer interaction data was integrated into the scene to drive the dynamic updating of the virtual vision. The final 3D virtual vision presentation effect was demonstrated in Fig. 6. 


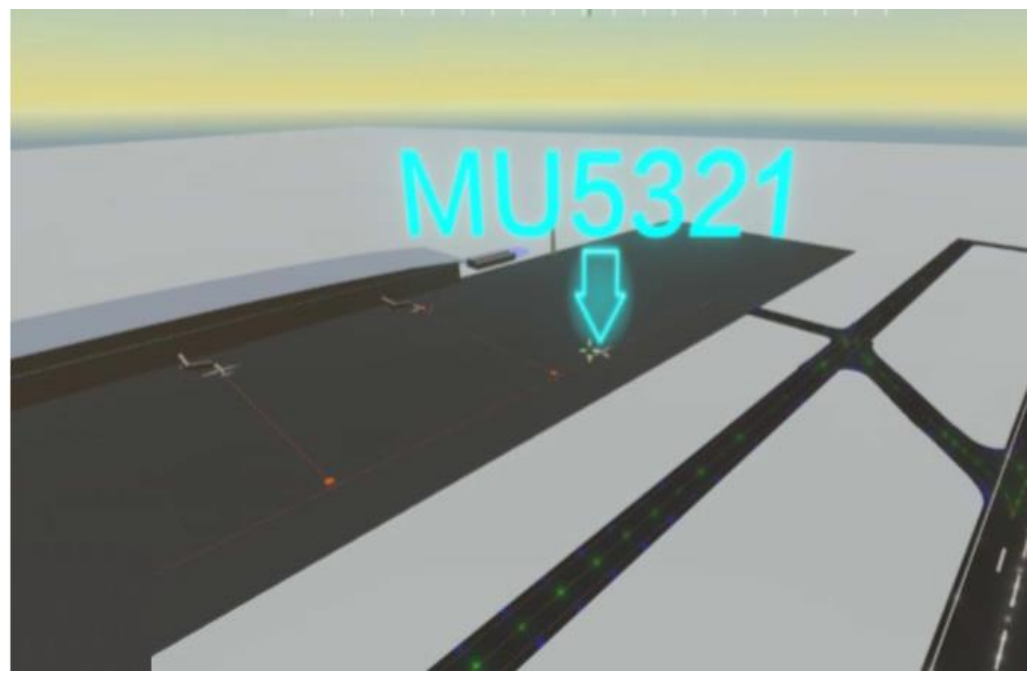

Fig. 6: 3D virtual vision of airport airside area

\subsection{Human-machine Interface Design of the Platform}

Based on the system functional requirements, the GUI module of Unity3D is used to design the basic human-machine interface, which mainly includes the function modules such as project management, model management, virtual simulation, and ATCT position sitting function package, and so on. We adopt a hierarchical UI interface design idea in the development process in order to support the centralized management of models for tower, runway, taxiway, aircraft, and other models of airside areas. And, for the good functional requirements of the platform, multi-thread technology is used to optimize the overall virtual vision rendering. The human-machine interface is demonstrated in Fig. 7.

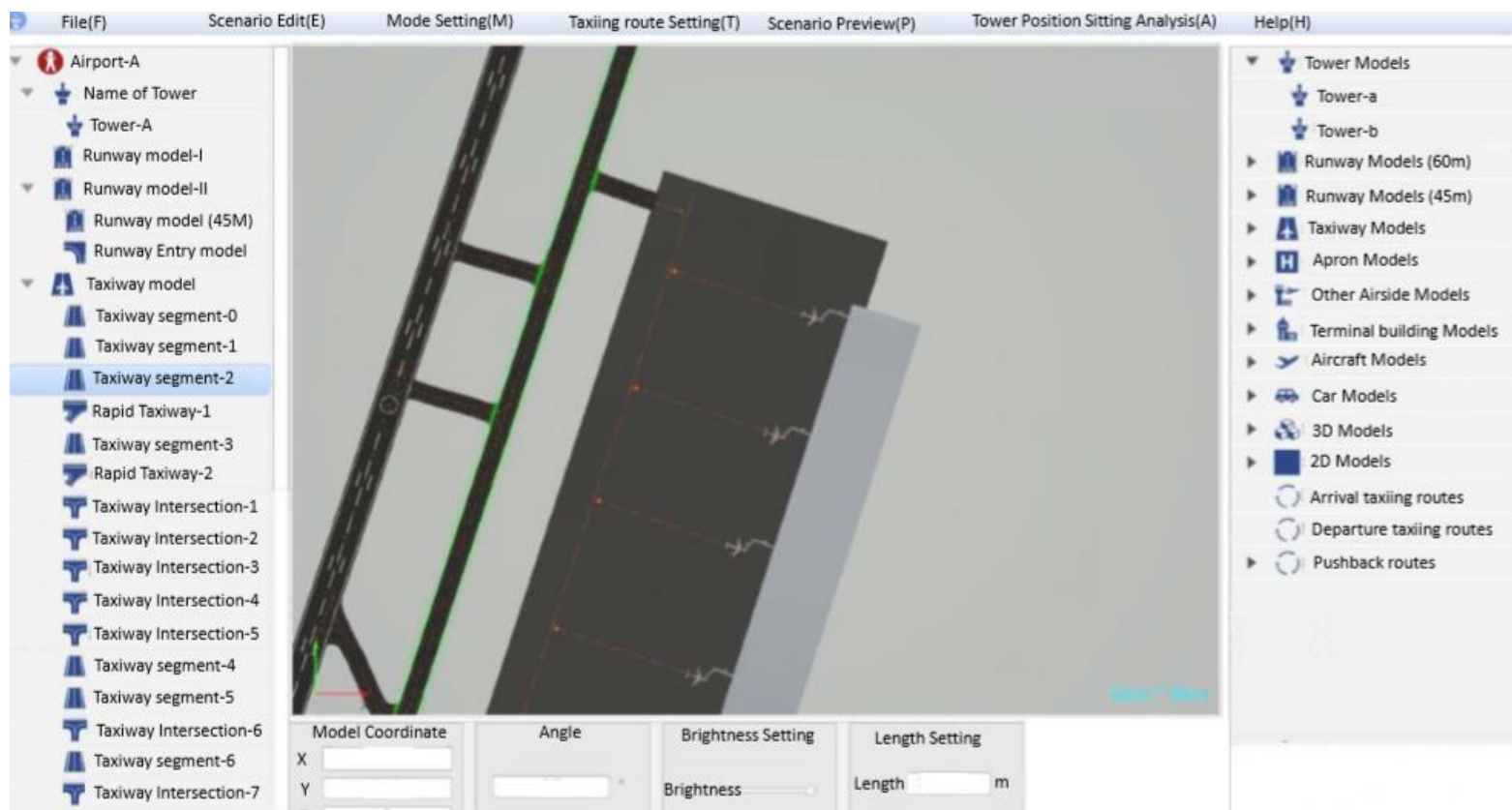

Fig. 7: Human-machine interface of the platform

\subsection{Design of ATCT Position Sitting Function Package}

The FAA has released an ATCT tower site selection criterion in FAA ORDER 6480.4A [11] in 2006, which includes tower site selection simulation. And, CAAC (Civil Aviation Administration of China) has published related document. As an illustration of the platform function, the ATCT tower position sitting function package is a plug-in algorithm module developed in the platform in this paper. We could conduct an evaluation on the line of sight form the prospective ATCT site to the airport movement area as well as to the airport building. Any sight obstructions caused by buildings can be demonstrated or be observed through VR helmet. The most feasible ATCT site options could be verified quickly and directly according to the established criteria and other relevant factors. The sight obstruction demonstration of ATCT is shown in Fig. 8. 


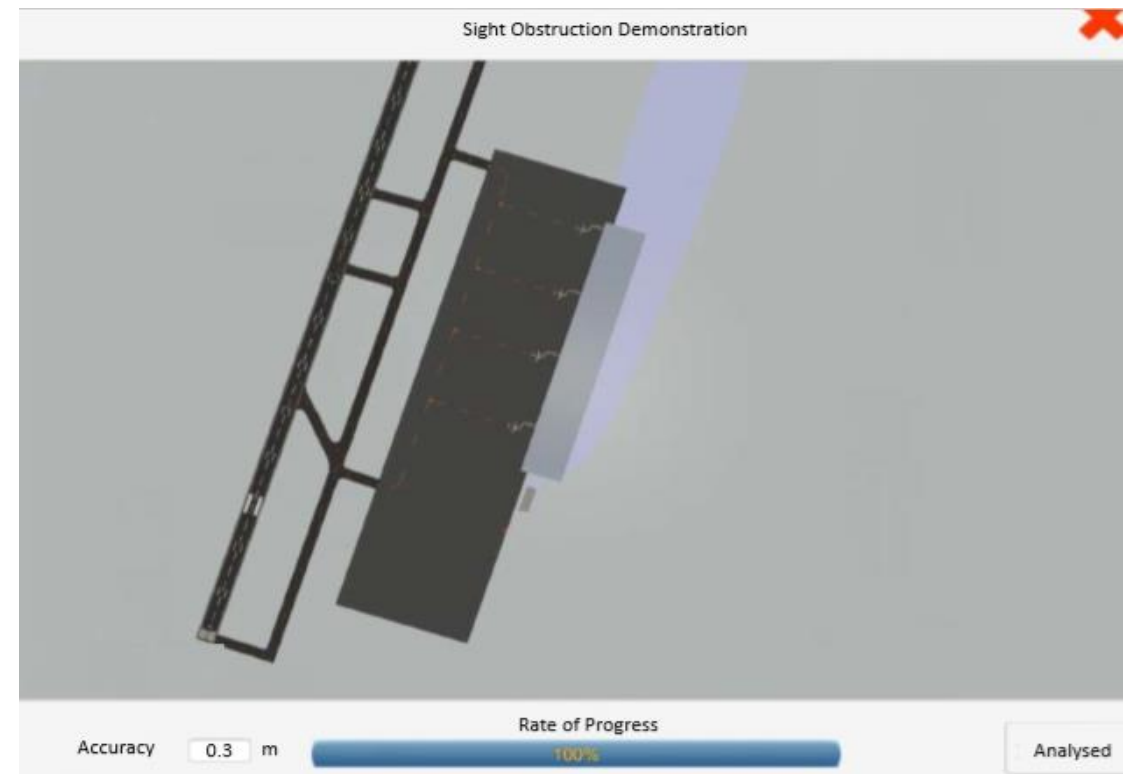

Fig. 8: Sight obstruction demonstration of ATCT by using plug-in function package in platform

\subsection{Hardware Implementation of Platform}

The platform runs on computer with high-performance image processing capabilities, and supports human-computer interaction based on the PC or with the help of a VR helmet and handle. The humancomputer interaction in the scene of the user is mainly reflected in as follows: on the one hand, the user can complete the roaming in the 3D virtual vision by moving with the aircraft or vehicle or based on the setting of the handle action; on the other hand, the user can complete the direct judgment on the ATCT sight obstruction. The operation of the 3D virtual vision platform is shown in Fig. 9.

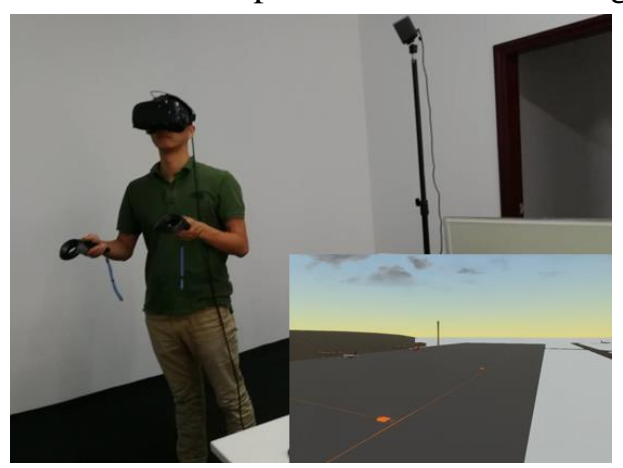

Fig. 9: The operation scene of the platform

\section{Platform Test}

In order to validate the practicability of the $3 \mathrm{D}$ virtual vision fast construction platform of the airport airside area, civil aviation airport designer were invited to test the function modules of the platform. The main test indicators include: 1) software friendly: for the user may not be a computer expert, the platform should be able to support users to get used to the software quickly, without remembering any instructions, but only the knowledge of airport airside design or operation; 2) Stability: The platform is design to producing the 3D virtual vision with large number of surface objects or buildings, and optimizing of the airport planning scheme. After nearly two months of test, the results indicated that the platform design is scientific, reasonable, simple and easy to use, and the functional modules are stable. At the same time, the plug-in tower position sitting function package is accurate and reliable.

\section{Conclusion}

A 3D virtual vision fast construction platform for airport design evaluation was developed based on VR technology. The platform integrates typical 3-dimensional model related to the airport airside system, including static traffic system models and movement objects. Users can quickly and efficiently create their own airport 3D model by select the model or modify the parameter of models. The VR technology used in 
this platform provides an intuitive means to verify the airport design schema before airport building or expansion. And the architecture of this platform supports the dynamically import new models or plug-in function packages. As a demonstration, we have developed an ATCT position sitting function package and worked well. In the future, we will develop much more function algorithms relevant to airport building or operation schema.

\section{Acknowledgements}

The authors would like to thank the anonymous reviewers whose comments have helped us to improve the presentation of the paper. This research is supported by Sichuan Science and Technology Program (Grant No.2020YFS0541), Fund for Young Scholars of CAFUC (Q2015-079), and Joint Funds of the National Science Foundation of China and the Civil Aviation Administration (Grant No. U1733105).

\section{References}

[1] Q. Zhao. Ten scientific and technical problems in virtual reality (in Chinese). Sci Sin Inform,2017,47:800-803.

[2] K. Yan, L. Tian, T. Liu. Design of aviation flight airworthiness support simulation training system based on VR. Modern Electronic Technique, 2019,42(1):134-138.

[3] Y. Gao, X. Zhong, Z. Xu. Virtual teaching experiment system of aero-engine based on VR. Journal of System Simulaition, 2008,20(11):2925-2929.

[4] S. Tia, W. Liu, H. Zhang. Research of VR-based 3D NC maching environment and key technology. Journal of System Simulaition, 2007,19(16):3727-3730.

[5] H. Gong, X. Zhou. Flight short-term collision detection based on control tower simulation system.Computer Technology and Development.2013,23(4):151-153.

[6] N. Cheng, P. Tuo. Research and simulation on key technologies of airliner's flight management system under next-generation navigation system (in Chinese).Sci Sin Tech,2018,48(3):264-276.

[7] D. Trenholme, S. Smith. Computer game engines for developing first-person virtual environments. Virtual Reality,2008,12: 181-187.

[8] D. Trenholme, S. Smith. Rapid prototyping a virtual fire drill environment using computer game technology.Safety Journal, 2009,5: 559-569.

[9] M. Dennis, H. Doug. UnrealTriage: A Game-Based Simulation for Emergency Response.report, 2012.

[10] M. Dennis, H. Amy. A Simple Distributed Simulation Architecture for Emergency Response Exercise,report 2013.

[11] FAA. Airport traffic control tower sitting process(6480.4A). 2006, April. 